\title{
CONSTRUCCIÓN Y VALIDACIÓN DE UN CUESTIONARIO PARA MEDIR CONDUCTAS Y HÁBITOS ALIMENTARIOS EN USUARIOS DE LA ATENCIÓN PRIMARIA DE SALUD
}

\author{
DEVELOPMENT OF A QUESTIONNAIRE \\ TO MEASURE BEHAVIOR AND FOOD HABITS IN \\ USERS OF PRIMARY HEALTH CARE
}

\author{
José Luis Pino V. (1), Claudio Díaz H. (2), Miguel Ángel López E. (2) \\ (1) Carrera Nutrición y Dietética, Universidad del Mar, Sede Centro Sur. Talca, Chile. \\ (2) Departamento de Investigación, Capacitación y Perfeccionamiento, \\ Universidad del Mar, Sede Centro Sur. Talca, Chile.
}

\begin{abstract}
To identify behaviors and habits of the population is critical to establish appropriate strategies and tools to promote changes inappropriate practices. The aim of this study was to develop a reliable tool to quantify and determine eating habits both in individuals and in the population. Based on the survey validated by Pardo in 2004, a group of students of 3rd year of Nutrition and Dietetics took a survey of 44 items, which was applied to a sample of 402 subjects (220 women and 180 males) whose average age was $30.2 \pm 24.7$ years. The questionnaire showed acceptable reliability $(\alpha-C r o n b a c h=0.792)$, establishing 11 dimensions with 38 questions. Females showed a significantly higher average total score compared to males $(p<0.001)$. In conclusion, implementation and validation of the questionnaire allows us to contribute a low-cost tool to know the intimate aspects of the behavior of people attending public health centers.
\end{abstract}

Key words: Validation studies, nutrition surveys, food habits, health centers, primary health care.

Este trabajo fue recibido el 21 de Junio de 2010 y aceptado para ser publicado el 27 de Enero de 2011.

\section{INTRODUCCIÓN}

El mundo moderno en el cual vivimos, concatenado fuertemente desde el prisma económico, y por otro lado, por una sociedad posmodernista influenciada desde lo cultural; termina repercutiendo en la formación, estilos de vida y hábitos culturales de las poblaciones, los cuales determinan entre otros factores, profundos cambios en los hábitos alimentarios dignos de tratar desde la epidemiología (1).

A lo anterior, se suma que la falta de una mirada multidisciplinaria, hace necesario incentivar una visión más holística para la generación de la ciencia. Por lo tanto, es importante seguir buscando nuevos y mejores métodos para su análisis y, en especial, los requeridos para puntualizar la colaboración entre científicos de distintas áreas" (2).

Está descrito que los hábitos y conductas alimenta- rias tienen una relación directa con la morbimortalidad de la población (3) debido a las transformaciones vertiginosas que ha tenido el estilo de vida y la alimentación de las comunidades en desarrollo, incluyendo Chile (4), cambios que han derivado desde lo socioeconómico (5) y han ocasionado una enorme dificultad para tratar de determinar los hábitos y conductas alimentarias.

Debido a lo anterior la malnutrición por exceso ha aumentado sostenidamente durante los últimos años en todos los grupos etarios, tanto a nivel mundial como nacional $(6,7)$. La obesidad ha pasado de ser una "condición" que repercute en la salud, a una enfermedad y pandemia. Según las estimaciones de la Organización Mundial de la Salud, se estima para el año 2015 que en el mundo existirán aproximadamente 2.300 millones de adultos con sobrepeso y más de 700 millones con obesidad (8). 
Este desbalance energético que produce el almacenamiento y aumento de la masa adiposa corporal, surge de la combinación de diversos factores, tales como, tiempo frente a la televisión (9), publicidad de los alimentos $(10,11)$, factores económicos (12), actividad física (13), percepción de la imagen corporal (14), conductas saludables (15), sedentarismo (16), entre otros, siendo la mayoría de estas conductas y hábitos, factores ambientales modificables.

Los hábitos alimentarios han cambiado notablemente en los últimos años, no sólo en Chile, sino en toda Latinoamérica (17), destacando un bajo consumo de frutas, lácteos, pescados y un alto consumo de azúcares simples y grasas (18) en todos los grupos etarios (1921), cambio, que desató un disparo en el diagnóstico de enfermedades crónicas no transmisibles, considerando lo anterior en sectores con disponibilidad y acceso de alimentos.

Organizaciones internacionales y gobiernos, incluido el de Chile, han elaborado múltiples estrategias para afrontar el aumento de la malnutrición por exceso (22), programas como "5 al día", Guías Alimentarias, Guías de Actividad Física, Programa EGO y EGO escuelas, entre otras iniciativas han tratado de disminuir los niveles de sobrepeso y obesidad e incentivar buenos hábitos de alimentación y de actividad física.

Muchas de las estrategias educacionales se basan en intervenciones transversales, las cuales no tienen permanencia en el tiempo; talleres de alimentación saludable, consejerías, talleres de actividad física, entre otros, son implementados de forma parcelada teniendo un escaso efecto. Estudios demuestran que estas estrategias no duran más allá de 3 meses y en ese aspecto tienen un mejor impacto los descuentos en los alimentos saludables $(23,24)$.

La modificación de conductas o de hábitos no es susceptible de cambios por educaciones esporádicas. Para tratar de cambiar los hábitos de la población primero se debe conocer cuáles son, luego determinar qué tipo de conductas, costumbres y creencias son las que priman, de tal forma de poder intervenir con las estrategias y herramientas pertinentes para cada sector o grupos específicos de la comunidad.

Actualmente, existen cuestionarios para cuantificar y determinar diferentes condiciones, tales como trastornos de la conducta alimentaria $(25,26)$, estado nutricional y envejecimiento (27), hábitos de sobrepeso y obesidad (28), hábitos alimentarios y consumo de grasas y azúcares (29), conducta alimentaria (TFEQ) (30), entre otros. En este contexto se presenta el siguiente estudio que pretende construir un cuestionario tipo encuesta que mida de forma precisa y confiable los hábitos y conductas de una población típica, de forma tal que permita obtener una panóptica clara de la situación alimentaria-nutricional de la población en riesgo que asiste a la red asistencial primaria pública.

\section{MATERIALES Y MÉTODOS}

El estudio es de diseño no experimental de tipo transversal, tomando como base el cuestionario validado por Pardo (2004) (23), instrumento de recolección de datos creado específicamente para determinar hábitos de sobrepeso y obesidad en la población obesa española. Contempla en una primera etapa un total de 57 preguntas escala tipo Likert, las cuales se filtraron y se obtuvo un total de 22 preguntas agrupadas en 5 dimensiones. El cuestionario fue analizado por 15 estudiantes de $\mathrm{Nu}$ trición y Dietética de tercer año, restando y sumando nuevas preguntas para adaptarlo a la realidad nacional y local. El instrumento final, se sometió a validación a través de juicios expertos el cual fue realizado por un nutricionista y un sociólogo.

Se determinó un total de 48 preguntas, de las cuales; 33 fueron escala tipo Likert, 14 de selección múltiple con cinco alternativas y 1 de jerarquización. Sólo se consideraron como parte de este trabajo aquellas preguntas de escala tipo Likert y de selección múltiple.

De un total de 19.062 usuarios inscritos válidos pertenecientes al Centro de Salud Familiar "Las Américas" de Talca, se seleccionó una muestra con $5 \%$ de error y un 95\% de nivel de confianza, arrojando una muestra total de 377 sujetos, aumentando el (n) un 7\%, con el objeto de lograr mayor representatividad y validéz de la muestra.

Se aplicó el cuestionario a 402 personas de todos los grupos etarios, en el caso de menores de edad se consultó a su padre, madre o acompañante. En el caso de adultos mayores, se aplicó a sujetos autovalentes. El instrumento fue aplicado en el mismo centro de salud por estudiantes de tercer año de la Carrera de Nutrición y Dietética de la Universidad del Mar, Sede Centro Sur, campus Talca, bajo la tutela y supervisión del docente encargado de la asignatura Metodología de la Investigación.

\section{Análisis estadístico}

Para describir las variables intervalares se usó la media aritmética y desviación estándar, previo análisis exploratorio de los datos utilizando la prueba de ShapiroWilk, que arroja normalidad de un valor de $\mathrm{p}>0,05$. Para aquellas variables ordinales o nominales se las presentó por medio de tablas de contingencia.

La estructura matricial se construyó a través de un análisis factorial usando como método de extracción al análisis de Componentes Principales, con una solución rotada aplicando la metodología Varimax. Las dimensio- 
nes se construyeron teniendo en cuenta una saturación mayor a 1,0. El análisis de consistencia interna total y por dimensión se realizó mediante la aplicación del coeficiente alpha de Cronbach. Para estudiar la interrelación de las preguntas que conforman las dimensiones en relación al puntaje de la dimensión y del total del puntaje, se utilizaron coeficientes de correlación de Pearson.

Para determinar los puntos de corte del puntaje resultante del instrumento, se utilizaron los valores de percentil 25 , entre percentil 25 y percentil 75 y mayor a percentil 75. Para comparar los puntajes respecto a género y grupos de edad, se aplicaron las pruebas de tStudent y ANOVA de una vía, respectivamente. Se usó el programa estadístico SPSS 17.0.

\section{RESULTADOS}

Participaron 402 usuarios del Centro de Salud Familiar "Las Américas" de la ciudad de Talca, región del Maule, de los cuales el 44,8\% lo constituyeron varones $(\mathrm{n}=180)$. Al momento de la investigación se observó una edad media de 30,24 \pm 24,75 años, quienes están distribuidas en los distintos programas que el Ministerio de Salud ofrece a través del centro de salud.

Se recomienda un análisis factorial si el estadístico Kaiser-Meyer-Olkin (KMO) arroja un valor mayor a 0,5, siendo óptimo si el KMO fuera mayor a 0,7 adicionando la prueba de esfericidad de Bartlett significativo, que permite rechazar la hipótesis de matrices similares, existiendo una correlación entre los datos. Es así como el valor de KMO fue de 0,805 y la prueba de esfericidad de Bartlett fue un valor significativo con valor $\alpha<0,05$ $\left(\mathrm{Chi}^{2}=4569,021 ; 703 \mathrm{gl} ; \mathrm{p}<0,001\right)$; por tanto, se puede realizar un análisis factorial, que permite agrupar en factores comunes entre ellas, que llevan como nombre "dimensiones", quienes se conformaron mediante el método de Varimax.

Al evaluar la fiabilidad de las preguntas que formaron parte del análisis factorial, la tabla 1 indica que el alpha de Cronbach es adecuado ( $\alpha$-Cronbach $=0,792$ ). Las preguntas lograron construir una matriz con 11 dimensiones, quienes logran explicar un $60,68 \%$ de la varianza. Hubo una dimensión formada por una pregunta y tres con valores de alpha de Cronbach menor a 0,500. Lo anterior implica mencionar que no pueden aplicar las dimensiones por separado, ya que, la consistencia interna es mejor con todas las preguntas.

$\mathrm{Al}$ estudiar las correlaciones de cada una de las preguntas respecto a su dimensión y al total del instrumento, se observa que están correlacionadas significativamente, a excepción de las preguntas 24-r (dimensión 6) y la 22-r (dimensión 11), con el ítem total. Al estudiar las relaciones entre las dimensiones formadas por las preguntas de hábitos alimentarios, la tabla 2 muestra que la mayoría se correlaciona significativamente, aunque las dimensiones 3, 4 y 9 fueron las que menos se correlacionaron con el resto. Aunque fueron marcadas como significativas, es necesario recalcar que las preguntas 37-r de la dimensión 3, la pregunta 20 de la dimensión 4, las preguntas 12-r y 28 -r de la dimensión 6, la pregunta 9-r de la dimensión 9 y la pregunta 42 de la dimensión 9 presentaron correlaciones respecto al total del puntaje con valores de $r$ inferior a 0,200 . No existen correlaciones menores a 0,200 si se le compara las preguntas con el total de cada dimensión.

La tabla 4 muestra el nombre propio (etiqueta) para cada una de las dimensiones lograda por el análisis factorial, que viene a englobar en un concepto las preguntas que la forman parte. Además, en ella se observa la manera en que se construyó la escala que permitirá clasificar a los usuarios de la atención primaria a quien se le aplique este cuestionario, en la cual se debe sumar las respuestas a las preguntas que pertenezcan a cada una de las 11 dimensiones, quienes a cada sumatoria se le deberá multiplicar un factor, que da el peso que representa la dimensión en el cuestionario. Luego, se suman estas ponderaciones y se obtiene el puntaje final de ella. Su normalidad se estudió aplicando la prueba de Shapiro-Wilk a los puntajes totales obtenidos en la misma muestra, indicando la existencia de normalidad ( $>0,05)$, con lo cual la escala se puede estudiar bajo estadística paramétrica. Es así como se observó un puntaje promedio de 11,52 $\pm 1,78$ puntos, con valores mínimos y máximos de 7,24 y 16,87 puntos, respectivamente.

El puntaje total de las 11 dimensiones del instrumento, permite clasificar de acuerdo a tres puntos de corte. Es así como aquellos que tengan menos de 10,25 puntos, se les clasificará con hábitos alimentarios deficientes. Quienes presenten un puntaje dentro del rango entre los 10,25 y 12,55 puntos, se les considerará con hábitos alimentarios adecuados. Mientras quienes presenten un puntaje superior a 12,55 puntos, se les catalogará como personas con hábitos alimentarios saludables.

\section{DISCUSIÓN}

Desde la más prematura infancia, es necesario e importante el cuidado nutricional óptimo de niñas y niños, la cual posee relevancia desde la propia gestación de los sujetos. A partir desde la vida intrauterina se condiciona la aparición de factores de riesgo que se manifiestan en la adolescencia y adultez (31-32), favoreciendo el desarrollo de patologías crónicas como enfermedades cardiovasculares, hipertensión arterial, la obesidad y la diabetes tipo 2 (33). Efectivamente, con el correr de los tiempos y entender los fenómenos desde la 


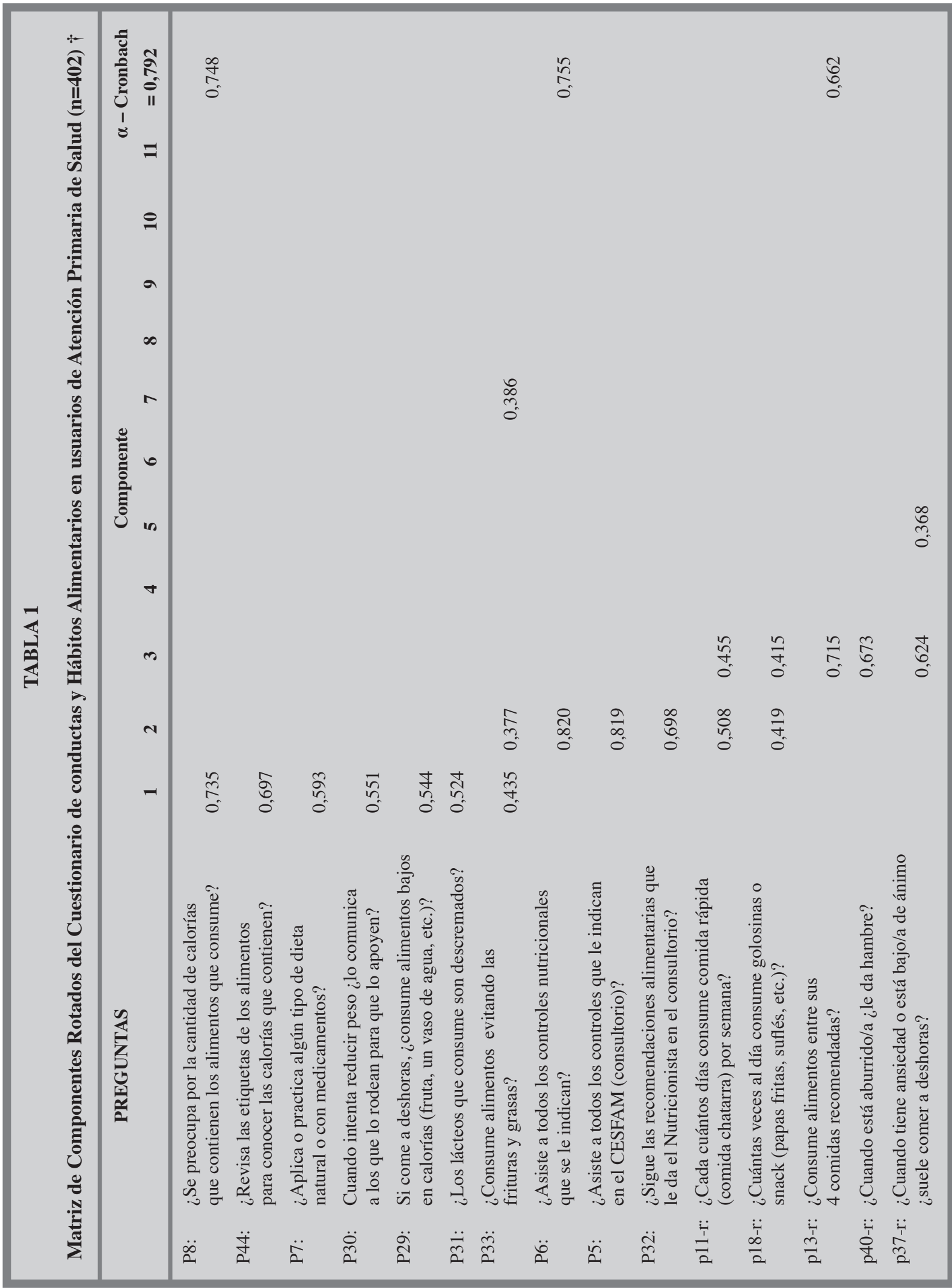




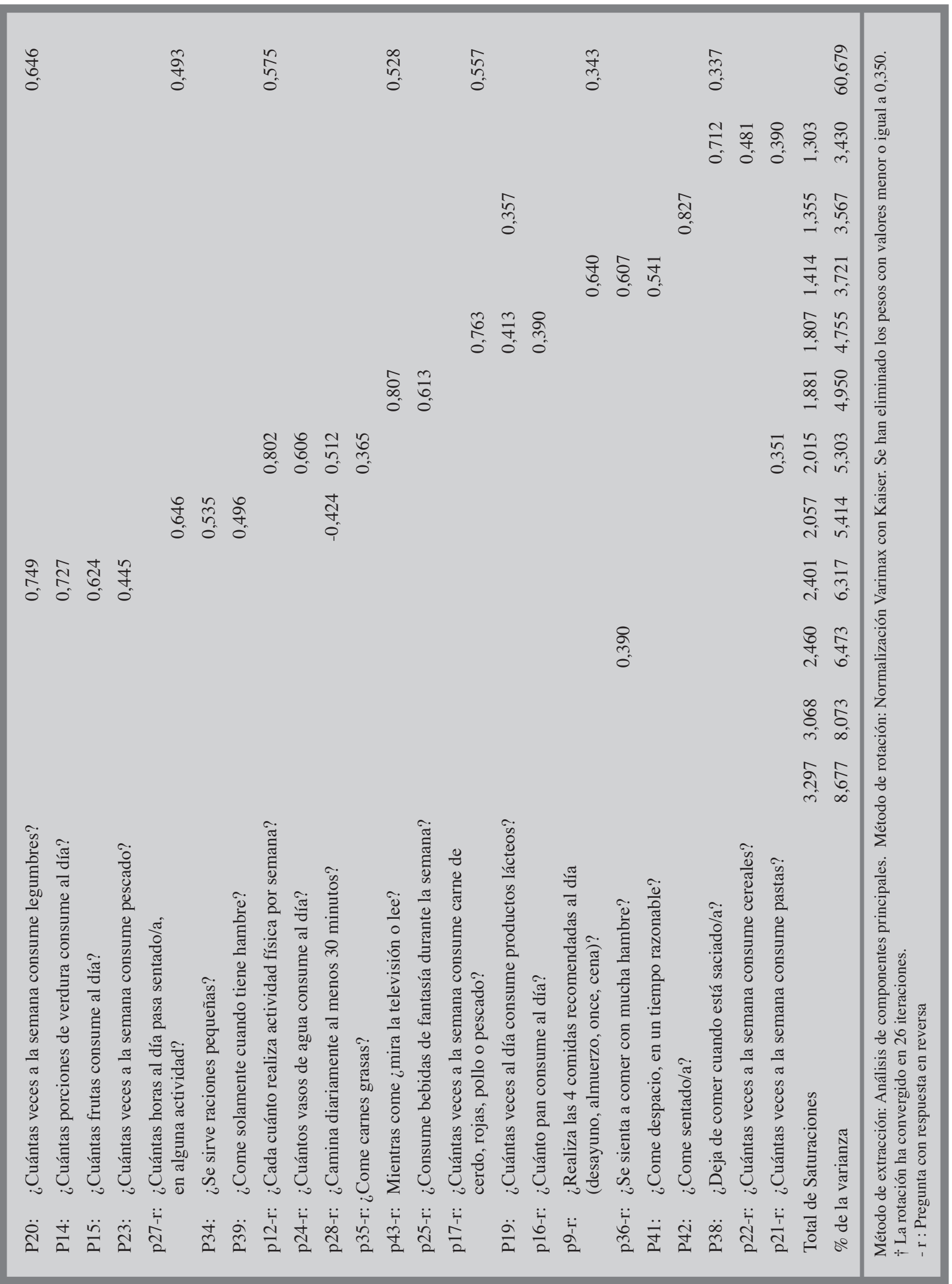


sociedad del riesgo, el interés de la nutrición poblacional es fundamental, ya que, está relacionado con los tipos de sociedad, cultura, costumbres, modelos económicos y políticos imperantes, los cuales determinarán la conducta, forma de estilos vida, e identidad del los sujetos. La literatura apunta como factores de elección de los alimentos el atractivo sensorial, costes de la salud, comodidad de adquisición y preparación, control de peso, conocimiento de alimento, regulación del humor, contenido natural de la comida y preocupaciones éticas (26).
Es cada vez más frecuente el impulso que se le ha dado a la alimentación y nutrición. Con los cambios propios de la industrialización se ha hecho una vinculación con el área de las ciencias sociales, ya que, la visión propia y holística desde la sociología, propone un método de análisis que nos lleva a entender el fenómeno de la mal nutrición, como un proceso dialéctico y socio cultural, que va a determinar al alero de lo fisiológico y biológico, formas de entender al sujeto desde su propia alimentación. Es así, que la creación de cuestionarios bajo esta

\section{TABLA 2}

\section{Correlaciones de Pearson Inter - Ítem de las dimensiones del cuestionario de conductas y Hábitos Alimentarios aplicado a usuarios de la atención primaria de salud $(n=402)$}

\begin{tabular}{|c|c|c|c|c|c|c|c|c|c|c|c|c|}
\hline & & $\operatorname{dim} 1$ & $\operatorname{dim} 2$ & $\operatorname{dim} 3$ & $\operatorname{dim} 4$ & $\operatorname{dim} 5$ & $\operatorname{dim} 6$ & $\operatorname{dim} 7$ & $\operatorname{dim} 8$ & $\operatorname{dim} 9$ & $\operatorname{dim} 10$ & $\operatorname{dim} 11$ \\
\hline \multirow{2}{*}{\multicolumn{2}{|c|}{$\operatorname{dim} 1 \mathrm{r}$}} & 1 & $0,345^{* *}$ & 0,089 & $0,346 * *$ & $0,404 * *$ & $-0,183 * *$ & $0,174 * *$ & $0,175^{* *}$ & $0,170^{* *}$ & $-0,006$ & 0,018 \\
\hline & $\mathrm{p}$ & & $<0,001$ & 0,076 & $<0,001$ & $<0,001$ & $<0,001$ & $<0,001$ & $<0,001$ & 0,001 & 0,900 & 0,714 \\
\hline \multirow{2}{*}{$\operatorname{dim} 2 \mathrm{r}$} & $\mathrm{r}$ & & 1 & $0,104^{*}$ & $0,162 * *$ & $0,316^{* *}$ & 0,044 & $0,377 * *$ & $0,415^{* *}$ & $0,131^{* *}$ & 0,088 & $0,217 * *$ \\
\hline & $\mathrm{p}$ & & & 0,037 & 0,001 & $<0,001$ & 0,382 & $<0,001$ & $<0,001$ & 0,009 & 0,077 & $<0,001$ \\
\hline \multirow{2}{*}{$\begin{array}{r}\operatorname{dim} 3 r \\
r\end{array}$} & $\mathrm{r}$ & & & 1 & 0,073 & $0,349 * *$ & $-0,073$ & $0,186^{* *}$ & 0,069 & $0,264^{* *}$ & 0,029 & $0,160 * *$ \\
\hline & $\mathrm{p}$ & & & & 0,146 & $<0,001$ & 0,144 & $<0,001$ & 0,169 & $<0,001$ & 0,564 & 0,001 \\
\hline \multirow{2}{*}{$\begin{array}{rl}\operatorname{dim} 4 & \mathrm{r} \\
\mathrm{r}\end{array}$} & $\mathrm{r}$ & & & & 1 & $0,247 * *$ & $-0,190 * *$ & 0,032 & 0,081 & $-0,038$ & $-0,022$ & $-0,197 * *$ \\
\hline & $\mathrm{p}$ & & & & & $<0,001$ & $<0,001$ & 0,520 & 0,104 & 0,451 & 0,654 & $<0,001$ \\
\hline \multirow[t]{2}{*}{$\operatorname{dim} 5$} & $\mathrm{r}$ & & & & & 1 & $-0,055$ & $0,199 * *$ & $0,253^{* *}$ & $0,189^{* *}$ & $0,113^{*}$ & $0,206 * *$ \\
\hline & $\mathrm{p}$ & & & & & & 0,268 & $<0,001$ & $<0,001$ & $<0,001$ & 0,024 & $<0,001$ \\
\hline \multirow{2}{*}{$\begin{array}{rl}\operatorname{dim} 6 & \mathrm{r} \\
\mathrm{r}\end{array}$} & $\mathrm{r}$ & & & & & & 1 & $0,192 * *$ & $0,315^{* *}$ & $-0,097$ & 0,058 & $0,203 * *$ \\
\hline & $\mathrm{p}$ & & & & & & & $<0,001$ & $<0,001$ & 0,052 & 0,245 & $<0,001$ \\
\hline \multirow{2}{*}{$\begin{array}{rl}\operatorname{dim} 7 & r \\
& p\end{array}$} & $\mathrm{r}$ & & & & & & & 1 & $0,387^{* *}$ & 0,044 & 0,043 & $0,213 * *$ \\
\hline & $\mathrm{p}$ & & & & & & & & $<0,001$ & 0,382 & 0,389 & $<0,001$ \\
\hline \multirow[t]{2}{*}{$\operatorname{dim} 8$} & $\mathrm{r}$ & & & & & & & & 1 & $-0,071$ & $0,172 * *$ & $0,258 * *$ \\
\hline & $\mathrm{p}$ & & & & & & & & & 0,154 & 0,001 & $<0,001$ \\
\hline \multirow[t]{2}{*}{$\operatorname{dim} 9$} & r & & & & & & & & & 1 & 0,044 & 0,047 \\
\hline & $\mathrm{p}$ & & & & & & & & & & 0,384 & 0,348 \\
\hline $\operatorname{dim} 10$ & & & & & & & & & & & 1 & $0,189 * *$ \\
\hline & $\mathrm{p}$ & & & & & & & & & & & $<0,001$ \\
\hline $\operatorname{dim} 11$ & & & & & & & & & & & & 1 \\
\hline & $\mathrm{p}$ & & & & & & & & & & & \\
\hline
\end{tabular}


perspectiva y áreas del conocimiento viene a comprender las conductas y proyectar acciones específicas que den resultados efectivos y eficaces.

Las complejas relaciones entre la salud, la dieta y patrones de los componentes han sido intensamente estudiados. Los investigadores han desarrollado diversos instrumentos, tales como los diarios de comidas y los cuestionarios de frecuencia alimentaria, para ayudar a comprender las relaciones entre los componentes de la dieta y la salud, y han desarrollado diversos indicadores tales como el índice de Alternativa de Alimentación Saludable, y la revisión del Índice de Calidad de la Dieta,

\section{TABLA 3}

Correlaciones de Pearson Inter-Dimensión, Ítem-Total y Dimensión-Total de la Estructura Factorial

\begin{tabular}{|c|c|c|c|c|c|}
\hline Preguntas & Ítem-dimensión & Ítem-total & Preguntas & Ítem-dimensión & Ítem-total \\
\hline P8 & $0,713 * *$ & $0,461 * *$ & $\mathrm{P} 12 \mathrm{r}$ & $0,728 * *$ & $0,114 *$ \\
\hline P44 & $0,694 * *$ & $0,449 * *$ & $\mathrm{P} 24 \mathrm{r}$ & $0,703 * *$ & 0,076 \\
\hline P7 & $0,572 * *$ & $0,306^{* *}$ & $\mathrm{P} 28 \mathrm{r}$ & $0,700 * *$ & $-0,125^{*}$ \\
\hline $\mathrm{P} 30$ & $0,559 * *$ & $0,245^{* *}$ & $\mathrm{P} 35 \mathrm{r}$ & $0,512 * *$ & $0,506^{* *}$ \\
\hline P29 & $0,630 * *$ & $0,307 * *$ & \multicolumn{2}{|c|}{ Total Dimensión 6} & $0,194 * *$ \\
\hline P31 & $0,677 * *$ & $0,473 * *$ & $\mathrm{P} 43 \mathrm{r}$ & $0,831 * *$ & $0,350 * *$ \\
\hline P33 & $0,572 * *$ & $0,619 * *$ & $\mathrm{P} 25 \mathrm{r}$ & $0,818 * *$ & $0,533 * *$ \\
\hline \multicolumn{2}{|c|}{ Total Dimensión 1} & $0,649 * *$ & \multicolumn{2}{|c|}{ Total Dimensión 7} & $0,531 * *$ \\
\hline P6 & $0,836 * *$ & $0,525^{* *}$ & $\mathrm{P} 17 \mathrm{r}$ & $0,733 * *$ & $0,326^{* *}$ \\
\hline P5 & $0,766^{* *}$ & $0,409 * *$ & P19 & $0,720 * *$ & $0,322 * *$ \\
\hline P32 & $0,797 * *$ & $0,637 * *$ & P16r & $0,731 * *$ & $0,585^{* *}$ \\
\hline $\mathrm{P} 11 \mathrm{r}$ & $0,585^{* *}$ & $0,525 * *$ & \multicolumn{2}{|c|}{ Total Dimensión 8} & $0,566 * *$ \\
\hline $\mathrm{P} 18 \mathrm{r}$ & $0,522 * *$ & $0,426 * *$ & P9r & $0,635 * *$ & $-0,199 * *$ \\
\hline \multicolumn{2}{|c|}{ Total Dimensión 2} & $0,705 * *$ & P36r & $0,702 * *$ & $0,359 * *$ \\
\hline $\mathrm{p} 13 \mathrm{r}$ & $0,692 * *$ & $0,356^{* *}$ & $\mathrm{P} 41$ & $0,639 * *$ & $0,505^{* *}$ \\
\hline $\mathrm{p} 40 \mathrm{r}$ & $0,827 * *$ & $0,318 * *$ & \multicolumn{2}{|c|}{ Total Dimensión 9} & $0,323 * *$ \\
\hline $\mathrm{p} 37 \mathrm{r}$ & $0,793 * *$ & $0,296 * *$ & $\mathrm{P} 42$ & 1,000 & $0,163 * *$ \\
\hline \multicolumn{2}{|c|}{ Total Dimensión 3} & $0,417 * *$ & \multicolumn{2}{|c|}{ Total Dimensión 10} & $0,163 * *$ \\
\hline $\mathrm{P} 20$ & $0,726 * *$ & $0,123^{*}$ & P38 & $0,642 * *$ & $0,365^{* *}$ \\
\hline P14 & $0,756 * *$ & $0,230 * *$ & $\mathrm{P} 22 \mathrm{r}$ & $0,669 * *$ & $-0,003$ \\
\hline P15 & $0,741 * *$ & $0,339 * *$ & $\mathrm{P} 21 \mathrm{r}$ & $0,661 * *$ & $0,376^{* *}$ \\
\hline $\mathrm{P} 23$ & $0,551^{* *}$ & $0,249 * *$ & Total Dime & n 11 & $0,371 * *$ \\
\hline \multicolumn{2}{|c|}{ Total Dimensión 4} & $\mathbf{0 , 3 3 8 * *}$ & & & \\
\hline $\mathrm{P} 27 \mathrm{r}$ & $0,618 * *$ & $0,198 * *$ & & & \\
\hline P34 & $0,764 * *$ & $0,626 * *$ & \multicolumn{3}{|c|}{$* \mathrm{p}<0,05 ; * * \mathrm{p}<0,01$ (prueba bilateral) } \\
\hline P39 & $0,726^{* *}$ & $0,474 * *$ & & $\mathrm{n}=402$ casos & \\
\hline \multicolumn{2}{|c|}{ Total Dimensión 5} & $0,624 * *$ & & & \\
\hline
\end{tabular}


para ayudar a comprender las relaciones entre la dieta y la salud (34).

Es conocida la utilidad de los cuestionarios dietéticos, que indagan aspectos cuantitativos de la alimentación de los sujetos, en donde el método más frecuentemente utilizado para determinar la ingesta alimentaria corresponde al cuestionario de frecuencia alimentaria (CFA), que sumados a los registros dietéticos (DR) y el recordatorio de 24 horas son los métodos de referencia más usados. Al estudiar la correlación de la ingesta de minerales obtenido por el CFA versus el método de referencia, indicó una calificación aceptable (35). No obstante, este cuestionario fue creado con el objeto de indagar aún más los hábitos alimentarios, y no de un modo meramente alimentario, demostrando ser una herramienta que contribuye a evaluar las conductas y hábitos tanto de forma individual como poblacional, pudiendo ser replicado en poblaciones con el perfil que se tomó como base para su creación y aplicación diagnóstica, lo cual cobra importancia, dado que en la actualidad se utilizan métodos de evaluación de la dieta que a menudo presentan dificultades para los investigadores y los encuestados y errores de declaración de datos inexactos que son comunes (36), explicado principalmente porque el desarrollo de la moderna metodología necesaria para caracterizar con rigor la ingesta en individuos y poblaciones data de hace sólo dos décadas, cuando germinó la epidemiología nutricional (37).

Es así, como el cuestionario que se sometió a evaluación factorial contiene treinta y ocho de las 48 preguntas

\section{TABLA 4}

\section{Etiqueta y valores descriptivos del puntaje total del cuestionario de Conductas y Hábitos Alimentarios con Estructura Factorial en base a 11 dimensiones}

\section{DIMENSIÓN \\ ETIQUETA \\ $\mathbf{N}^{\circ}$ PREG \\ PONDERACIÓN}

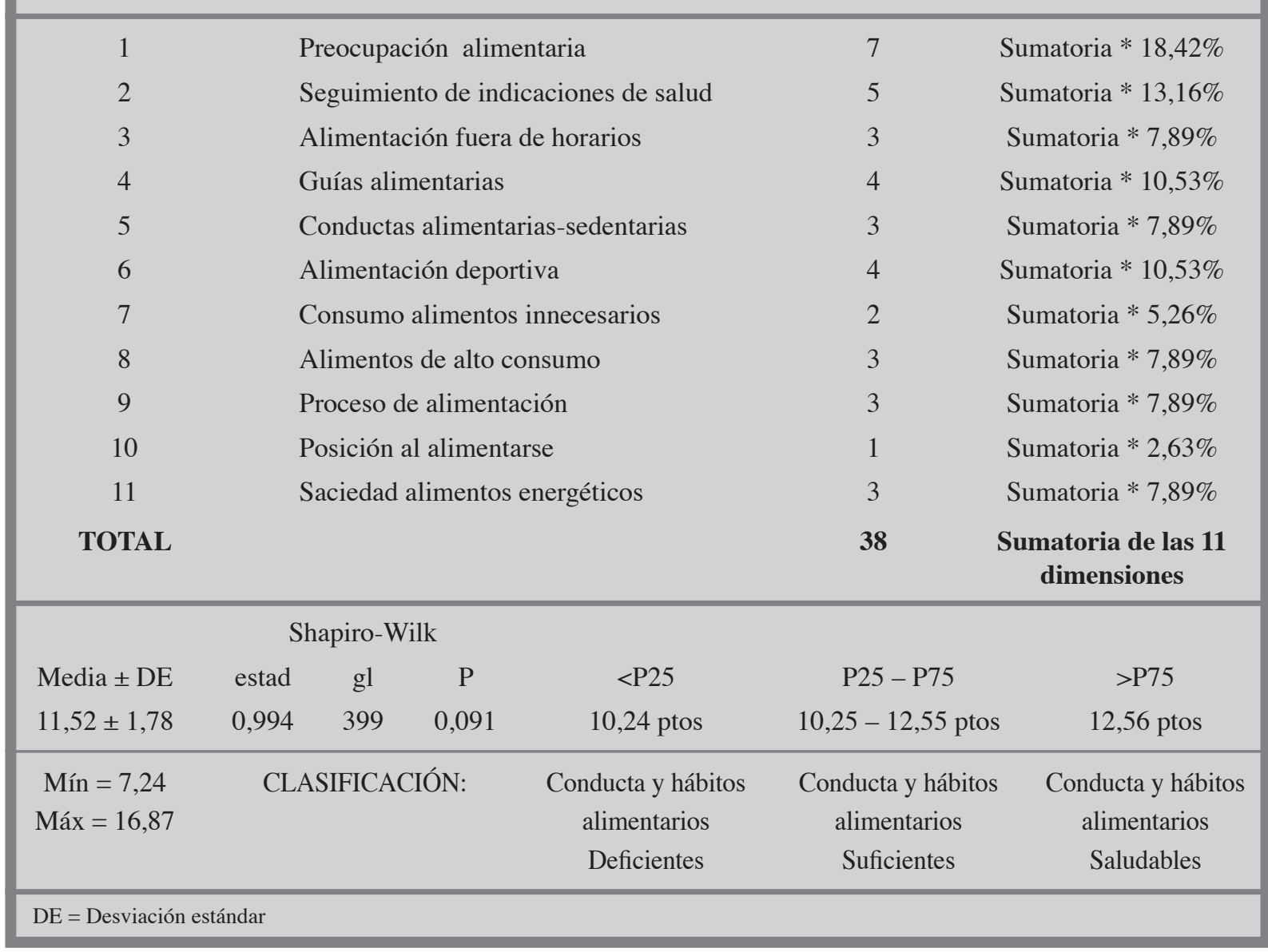


originalmente formuladas, detectándose 11 dimensiones; Preocupación alimentaria; que hace referencia al interés que muestran las personas por mantener un peso saludable y alimentarse saludablemente. Seguimiento de indicaciones de salud; identifica el nivel de compromiso tanto con los Centros de Salud como con la consulta nutricional y consecuentemente el seguimiento de indicaciones alimentarias saludables. Alimentación fuera de horarios: hace referencia al consumo de alimentos entre comidas asociado a conductas psicológicas. Guías alimentarias; trata de identificar en nivel de conocimiento que tienen las personas referentes a las guías alimentarias del Ministerio de Salud, orientado a los alimentos de menor consumo; frutas, verduras, pescado y legumbres. Conductas alimentarias-sedentarias; hace referencia al tiempo de inactividad durante el día y la asociación a un menor consumo de alimentos. Alimentación deportiva; se refiere a la actividad física realizada y la relación que existe con el consumo de ciertos alimentos que interfieren directamente en su realización, de forma positiva o negativa. Consumo alimentos innecesarios; agrupa 2 preguntas que revelan el consumo innecesario de calorías; viendo televisión y el consumo de bebidas de fantasía. Alimentos de alto consumo; hace referencia al consumo de carnes, lácteos y pan, alimentos que según las hojas de balance alimentario de la FAO han aumentado su consumo. Proceso de alimentación describe el número de comidas importantes que realiza una persona, la disposición y desarrollo del acto de alimentación. Posición al alimentarse; contempla solo una pregunta que es homónima. Saciedad alimentos energéticos; que hace referencia al consumo de alimentos que contienen hidratos de carbono complejos y el poder o nivel de saciedad de los mismos.

Si bien, esta nueva estructura de recolección de información puede resultar tediosa al encuestado, contribuye a elaborar una panorámica más amplia centrada en la valoración de conductas, que sin lugar a dudas, es una de las preocupaciones centrales en el combate de enfermedades crónicas no transmisibles. Aunque existen muchos métodos para medir el entorno alimentario, esta área de investigación es todavía relativamente nueva, por lo que existen escasos intentos sistemáticos para cuantificar, comparar y contrastar, o bien, para informar sobre sus propiedades psicométricas (38). En este contexto, este trabajo es una contribución para la elaboración de nuevos instrumentos, con evaluaciones de su estructura factorial, consistencia interna y de la capacidad diagnóstica que puedan obtener, de manera tal, que ayuden a objetivar los hábitos alimentarios, aportando información para la comprensión de la casuística de las enfermedades crónicas no transmisibles.

\section{RESUMEN}

Identificar las conductas y hábitos de la población es fundamental para establecer las estrategias y herramientas pertinentes para promover el cambio de las prácticas inadecuadas. El objetivo de este estudio es elaborar una herramienta confiable que permita cuantificar y determinar los hábitos alimentarios tanto individuales como poblacionales. En base a la encuesta validada por Pardo (2004), un grupo de estudiantes de $3^{\circ}$ año de Nutrición y Dietética elaboró una encuesta de 48 ítems, la cual se aplicó a una muestra de 402 personas de todos los grupos etarios (220 mujeres y 180 varones), cuya edad promedio fue de 30,24 $\pm 24,75$ años. El cuestionario revela una confiabilidad aceptable ( $\alpha$-Cronbach $=0,792$ ), estableciéndose 11 dimensiones con 38 preguntas. Las mujeres presentaron un puntaje total promedio significativamente superior en comparación a los varones $(\mathrm{p}<0,001)$. En conclusión, la aplicación y validación del cuestionario nos permite contribuir con una herramienta de bajo costo que permita conocer aspectos más íntimos de las conductas alimentarias que forman parte de los hábitos de personas que asisten a centros de salud públicos.

Palabras clave: Estudios de validación, encuestas nutricionales, hábitos alimenticios, centros de salud, atención primaria de salud.

Dirigir la correspondencia a:

Sr. José Luis Pino Villalón
Director Carrera Nutrición y Dietética
Universidad del Mar,
Sede Centro Sur
4 Poniente 1223
Talca, Chile.
Fono: (71) 417000 - 417035
Fax: (71)417062
E-mail: jose.pino@udelmar.cl

Agradecimientos: A las y los estudiantes del curso de Metodología de la Investigación, del año 2009, del tercer año de la carrera de Nutrición y Dietética de la Universidad del Mar, Sede Centro Sur, por su entusiasmo en el proceso de "aprender haciendo" y a la Sra. Berta Pinto Lemus, Directora del Centro de Salud Familiar "Las Américas" de Talca.

\section{BIBLIOGRAFÍA}

1. Moisés, Goldbaum. Estilos de vida y modernidad, Consultado el 26 de enero de 2011. Disponible en: [http://www.idrc.ca/es/ev-23071-201-1-DO_TOPIC.html]

2. Russell, Jane M, Ainsworth, Shirley y NarváezBerthelemot, Nora. Colaboración científica de 
la Universidad Nacional Autónoma de México (UNAM) y su política institucional. Rev Española Documentación Cient, 2006; (1), p 72.

3. Christina-Maria Kastorini, Haralampos J. Milionis, John A. Goudevenos, and Demosthenes B. Panagiotakos, Modelling the Role of Dietary Habits and Eating Behaviours on the Development of Acute Coronary Syndrome or Stroke: Aims, Design, and Validation Properties of a Case-Control Study, Cardiology Research and Practice, vol. 2011, Article ID 313948, 7 pages. [online].

4. Mendoza V, Carolina; Pinheiro F, Anna Christina y Amigo C, Hugo. Evolución de la situación alimentaria en Chile. Rev Chil Nutr 2007, 34, (1), 62-70.

5. Espinosa Gonzalez, Leticia. Cambios del modo y estilo de vida; su influencia en el proceso saludenfermedad. Rev Cubana Estomatol 2004, vol.41, n.3 [online].

6. Popkin BM y Doak CM: The obesity epidemic is a Worldwide phenomenon. Nutr Rev, 1998, 56: 106-14.

7. Albala C, Vio F, Kain J, Uauy R. Nutrition transition in Chile: determinants and consequences. Public Health Nutr 2002; 5(1A):123-8.

8. Organización Mundial de la Salud, Obesidad y Sobrepeso, citado 13 de abril de 2010 Disponible en: [http://www.who.int/mediacentre/factsheets/ fs311/es/index.html]

9. Moreno L, Arturo Y Toro Z, Luis. La Televisión, Mediadora Entre Consumismo Y Obesidad. Rev Chil Nutr. 2009, 36, (1), 46-52.

10. Jennifer Culp, Robert A. Bell, Diana Cassady, Characteristics of Food Industry Web Sites and “Advergames" Targeting Children, J Nutr Educ Behavior, 2010; 42 (3) 197-201.

11. Olivares S, Yanez R, Diaz N. Publicidad de Alimentos Y Conductas Alimentarias En Escolares De $5^{\circ} \mathrm{A} 8^{\circ}$ Básico. Rev Chil Nutr 2003; 30 (1) 36-42.

12. Araya B, Marcela Y Atalah S., Eduardo. Factores Que Determinan La Selección De Alimentos En Familias De Sectores Populares. Rev Chil Nutr 2002; 29 (3) 308-15.

13. Caballero, C., Hernández, B., Moreno, H., Hernández, G. C., Campero, L., Cruz, A. y Lazcano, P. E. (2007). Obesidad, Actividad E Inactividad Física En Adolescentes De Morelos, México: Un Estudio Longitudinal. Arch Latinoam Nutr. 57(3): 231-37.

14. Pino V, José L., Lopez E, Miguel A, Moreno V, Alicia A. y Faundez P, Teresita del Pilar. Percepción de la imagen corporal del estado nutricional y de la composición corporal de estudiantes de nutrición y dietética de la Universidad del Mar, Talca, Chile.
Rev Chil Nutr 2010; 37 (3) 321-8.

15. López E, Miguel Ángel. Etapas Del Cambio Conductual Ante La Ingesta De Frutas Y Verduras, Control De Peso Y Ejercicio Físico De Estudiantes De La Universidad Del Desarrollo, Sede Concepción, Chile. Rev Chil Nutr 2008;35 (3) 215-24.

16. Buhring B, Kristian; Oliva M, Patricio Y Bravo C, Claudio. Determinación No Experimental De La Conducta Sedentaria En Escolares. Rev Chil Nutr 2009; 36 (1) 23-30.

17. Bermúdez OI, Tucker KL. Trends in dietary patterns in latin american populations. Cad Saüde Publica,(Río de Janeiro) 2003; 19 (supl):S87-S99.

18. Organización De Las Naciones Unidas Para La Agricultura Y La Alimentación (FAO), Faostat, Consultado el 14 abril de 2010. Disponible en: $<$ http://faostat.fao.org/site/291/default.aspx>

19. Zacarías H, Isabel et al. Consumo de verduras y frutas en centros de salud y supermercados, de la región metropolitana de Chile: Programa 5 al día. Rev Chil Nutr 2009; 36 (2)159-68.

20. MacMillan K, Norman. Valoración de hábitos de alimentación, actividad física y condición nutricional en estudiantes de la Pontificia Universidad Católica de Valparaíso. Rev Chil Nutr 2007; 34 (4)330-36.

21. Restrepo M, Sandra Lucia et al. Los hábitos alimentarios en el adulto mayor y su relación con los procesos protectores y deteriorantes en salud. Rev Chil Nutr 2006; 33 (3) 500-10.

22. Molina, Verónika. Guías Alimentarias en América Latina: Informe de la consulta técnica regional de las Guías Alimentarias. An Venez Nutr 2008; 21 (1) $31-41$.

23. Marjorie R. Freedman, Audrey Nickell, Impact of After-school Nutrition Workshops in a Public Library Setting, J Nutr Educ Behavior 2010; 42 (3), 192-6.

24. Ni Mhurchu C, Blakely T, Jiang Y, Eyles HC, Rodgers A., Effects of price discounts and tailored nutrition education on supermarket purchases: a randomized controlled trial, Am J Clin Nutr. 2010; 91(3):736-47.

25. Rueda, German Eduardo et al. Validación de la encuesta SCOFF para tamizaje de trastornos de la conducta alimentaria en mujeres universitarias. Biomédica. 2005; 25 (2) 196-202.

26. Alfonso Urzúa M., Sandy Castro R., America Lillo O., Carolina Leal P., Evaluación de los trastornos alimentarios: Propiedades psicométricas del test Edi-2 en adolescentes escolarizados(As) de 13 A 18 Años, Rev Chil Nutr 2009; 36 (4) 1063 - 73.

27. Cuyac Lantigua, Magdalena Y Santana Porben, Ser- 
gio. La Mini Encuesta Nutricional del Anciano en la práctica de un Servicio hospitalario de Geriatría: Introducción, validación y características operacionales. ALAN 2007, 57 (3) 255-65.

28. A. Pardo, M. Ruiz, E. Jódar, J. Garrido, J. M. De Rosendo, L. A. Usán, Desarrollo de un cuestionario para la valoración y cuantificación de los hábitos de vida relacionados con el sobrepeso y la obesidad, Nutr Hosp. (2004) XIX (2) 99-109.

29. Ana Gladys Aráuz Hernández, Marlene Roselló Araya, Sonia Guzmán Padilla, Gioconda Padilla Vargas, Validación de un cuestionario de hábitos alimentarios asociados al consumo de grasas y azúcares Arch Latinoam Nutr. 2008; 58 (4) 392-6.

30. Stunkard AJ y Messick S: The Three factor Eating Questionnaire to measure dietary restraint, disinhibition and hunger. J Psychosom Res, 1985, 29:71-83.

31. Tamashiro KL, Moran TH., Perinatal environment and its influences on metabolic programming of Offspring, Physiol Behav. 2010; 14;100(5):560-6.

32. Thornburg KL, O'Tierney PF, Louey S., Review: The placenta is a programming agent for cardiovascular disease, Placenta. 2010;31 Suppl:S54-9
33. Rodríguez - Santos Francisco, Aranceta B. Javier, Serra M. Lluís, Psicología y nutrición, Elsevier masson, España, 2008. p 17-34.

34. Govig B, de Souza R, Levitan EB, Crookston D, Kestens Y, Mendivil CO, Mittleman MA. The eating assessment table-an evidence-based nutrition tool for clinicians: background, description, future of tool. Crit Pathw Cardiol 2009; 8(2):55-6, 57-62.

35. Lluis Serra-Majem. Dietary assessment methods for intakes of iron, calcium, selenium, zinc and iodine. British J Nutr;2009, 102:S38-S55.

36. Ngo J, Engelen A, Molag M, Roesle J, GarcíaSegovia P, Serra-Majem L. A review of the use of information and communication technologies for dietary assessment. Br J Nutr. 2009; 101 Suppl 2:S102-12.

37. Martín-Moreno JM, Gorgojo L. Assessment of dietary intake at the population level through individual questionnaires: methodological shadows and lights. Rev Esp Salud Publica. 2007; 81(5):507-18.

38. McKinnon RA, Reedy J, Morrissette MA, Lytle LA, Yaroch AL. Measures of the food environment: a compilation of the literature, 1990-2007. Am J Prev Med. 2009;36(4 Suppl):S124-33. 